\title{
Promotion of underutilised manual vacuum aspiration service in early pregnancy care
}

\begin{abstract}
The health and social care information centre reports that the early pregnancy loss accounts for over 50,000 admissions per annum in the UK. It is expected that this emotional workload will increase exponentially with increase in the maternal age, assisted conception and maternal comorbidities. NICE guideline clearly outlines various treatment options including manual vacuum aspiration (MVA) for the management of miscarriage. Though outpatient MVA has many advantages over inpatient surgical evacuation this service is not provided in many units. On the other hand it is not discussed enough despite availability of this service in some units. We identified the underutilisation of this service in our hospital and took steps to promote the service. We trained our early pregnancy staff in counselling and offering this service. We also designed patient information leaflet and a dedicated team to care for these women during and after the procedure. We re-audited our service since staff training and found that the uptake has increased from $3 \%(4 / 154$ patients) to $41 \%$ (28/68 patients). The patient feedback was quite positive about this service.
\end{abstract}

Volume 10 Issue 2 - 2019

\author{
Revathi D Krishnan,' Padma Manda² \\ 'Obstetrics and Gynaecology, County Durham and Darlington \\ NHS Foundation Trust, United Kingdom \\ 2Obstetrics and Gynaecology, South Tees Hospital, United \\ Kingdom
}

Correspondence: Revathi D Krishnan, Obstetrics and Gynaecology, County Durham and Darlington NHS Foundation Trust, Tel 07737066778, Email dkrevathi@gmail.com

Received: June 04, 2018 | Published: March 06, 2019

Keywords: manual vacuum aspiration, miscarriage, surgery, pregnancy loss, retained products of conception

Abbreviations: MVA, manual vacuum aspiration; Surgical EVAC, surgical evacuation; POC, products of conception; TOP, termination of pregnancy; RCOG, royal college of obstetrician and gynaecologist

\section{Introduction}

One in five pregnancies ends up in miscarriage, causing considerable distress to the family and the health care provider. Those who undergo the process of miscarriage already feel emotionally and psychologically drained. The longer the waiting time from the diagnosis of miscarriage to the surgical treatment the more the patient dissatisfaction would be. Thus improvement in the management of miscarriage is vital to reduce the incidence of associated psychological morbidity. In the current climate of practise, it is essential to ensure that the limited resources of the National Health Service are used to maximise health benefits for its users. The NICE guideline for the management of miscarriage recommends individualisation of the care and it can be conservative, medical or surgical method. ${ }^{1}$ The surgical treatment can be in the operating theatre under general anaesthesia (surgical evacuation, EVAC) or in an outpatient set up (Manual Vacuum Aspiration, MVA). ${ }^{2}$ MVA is also an option for first trimester termination of pregnancy and for the retained products after medical or surgical TOP. ${ }^{3,4}$ The single use MVA kit is as shown in Figure 1. Those patients who are stable and booked for surgical management by patient's choice are at risk of cancellation or postponement during the process of prioritisation for theatre space. MVA has the advantage of avoiding general anaesthetic risk, lesser complications and early recovery and comparable success rate to the surgical evacuation. ${ }^{5-7}$ Moreover, MVA reduces the patient waiting time for the surgical treatment by not requiring the theatre space. MVA is successful in almost $98 \%$ of cases and can be more when done under ultrasound guidance.

\section{Initial audit}

We looked at the surgical management of miscarriage done at south tees hospital in 2014. We had four MVA against 150 EVAC over the period of twelve months (Figure 2). The time period from booking surgical EVAC to the procedure ranged from 77 minutes to three days. On further analysis of this huge variation in the number of MVA and EVAC we found that majority of the early pregnancy staff weren't comfortable counselling the MVA option. Although consultants were available to provide the service the uptake was poor due to poor counselling, staff hesitance and less informed patient.

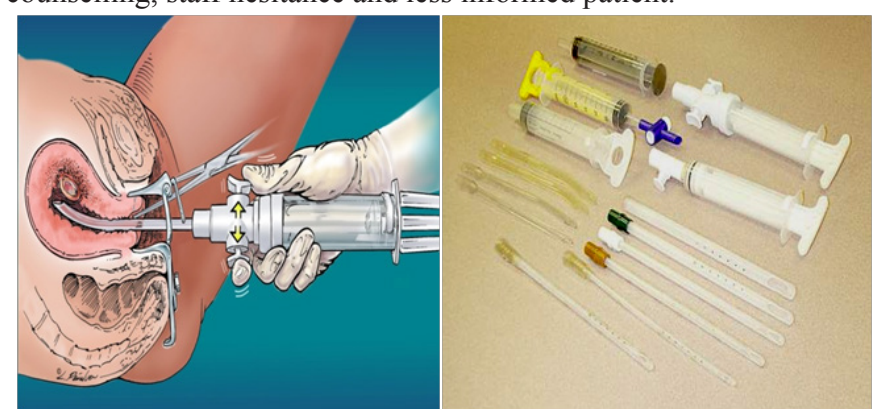

Figure I Single use MVA kit.

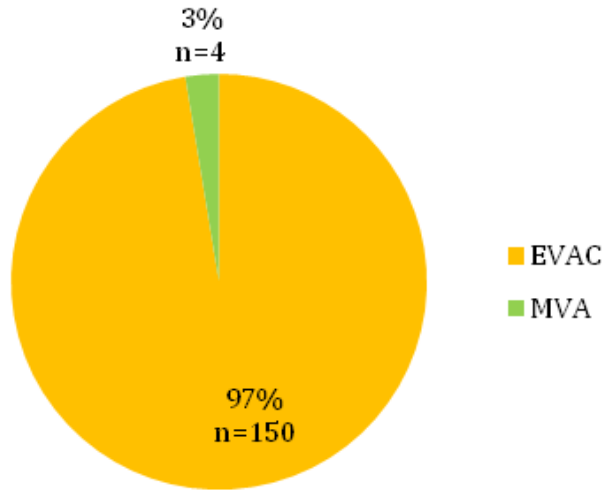

Jan - Dec 2014

Figure 2 Four MVA against I50 EVAC over the period of twelve months. 


\section{Promotion of the service}

A business plan was submitted and fund allotment to improve the MVA service was agreed on with the clinical commissioning group. A patient information leaflet and a care pathway for MVA were put in place. The care pathway includes booking patients for MVA in the acute gynaecology clinic which runs five days a week. The guideline was updated with the addition of MVA option for the management of miscarriage. A study session on counselling and technical training on MVA was arranged to early pregnancy staff and trainees. A separate register is maintained to audit the number and outcome of MVA including patient satisfaction since January 2015.

\section{Outcome}

We audited the MVA service since intense promotion. We looked at the number of MVA over a period of five months from February to June 2015. 28 out of 68 patients had MVA (Figure 3) and the various indications were as in Figure 4. The MVA uptake was improved from $4 \%$ to $41 \%$. 93\% (26/28) returned patient satisfaction survey and all were positive about the service and the team (Table 1).

Table I Patient satisfaction survey

1. $93 \%(26 / 28)$ returned the forms.

2. Friendly, helpful, supportive staff

3. Fabulous staff

4. Thank you for helping me to put an end to what has been a sad experience for us

5. Much better than going to sleep

6. An awful situation to go through but made more bearable

7. Lot easier than she thought

8. It was painful but didn't last long

9. Minor invasion of privacy

10. Scared to go home, would like to stay bit longer

\section{Feb - June 2015}

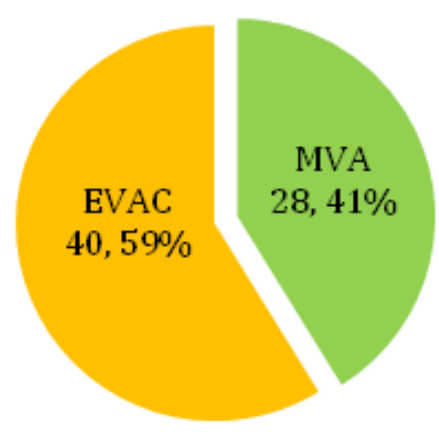

Figure 3 The number of MVA over a period of five months from February to June 2015 .

\section{Conclusion}

Though the MVA procedure is in practise for 30 years the service is not well recognised due to various reasons. It includes staff innocence, patient not given the option, not many clinicians have training to perform the procedure. Our project confirms that the success of the service is achieved by adequate staff training, appropriate patient counselling and dedicated team providing the service. The MVA is well recognised as a cost effective operator and patient friendly procedure. ${ }^{8}$ The RCOG conducts a study day for training and addressing issues around MVA to promote the underutilised MVA service. This is a simple but valuable service to be included in each and every early pregnancy unit.

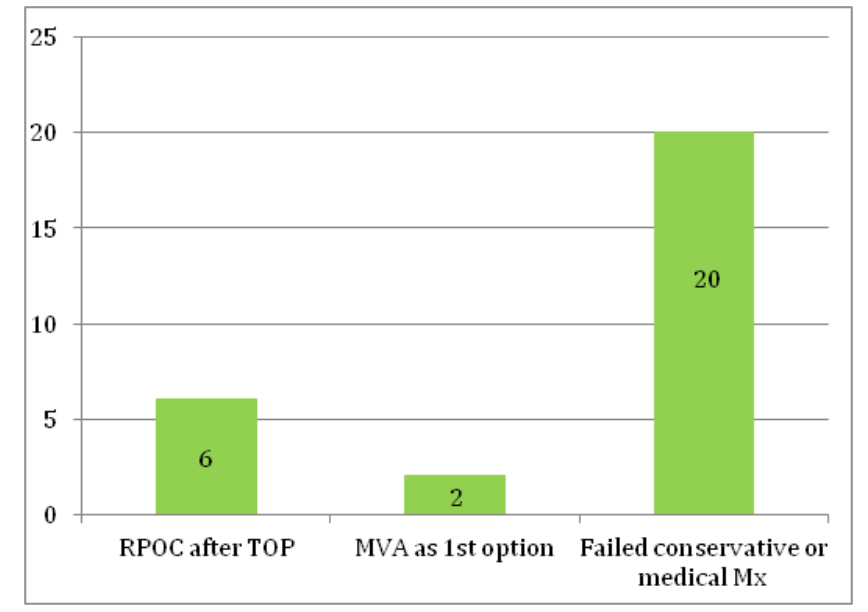

Figure 4 Various indications in MVA.

\section{Acknowledgments}

None.

\section{Conflicts of interest}

The authors declare no conflicts of interest.

\section{References}

1. CG 154 NICE Guideline. Ectopic pregnancy and miscarriage diagnosis and initial management. 2012.

2. Obs Gynae \& Midwifery News. MVA: an alternate method for uterine evacuation. 2012.

3. Yonke N, Leeman LM. First-trimester surgical abortion technique. Obstet Gynecol Clin North Am. 2013;40(4):647-670.

4. Goldberg AB, Dean G, Kang MS, et al. Manual versus electric vacuum aspiration for early first-trimester abortion: a controlled study of complication rates. Obstet Gynecol. 2004;103(1):101-107.

5. Morgan Leslie. The Case for Manual Vacuum Aspiration. Middle East Health (Durbin); 2012. 136 p.

6. Milingos D, Mathur M, Smith N, et al. Manual vacuum aspiration: a safe alternative for the surgical management of early pregnancy loss. BJOG. 2009;116(9):1268-1271.

7. Rausch M, Lorch S, Chung K, et al. A cost-effectiveness analysis of surgical versus medical management of early pregnancy loss. Fertil Steril. 2012;97(2):355-360.

8. Coombes R. Obstetricians seek recognition for Chinese pioneers of safe abortion. BMJ. 2008;336(7657):1332-1333. 